\title{
PSEUDO AFFINE WIGNER DISTRIBUTIONS
}

\author{
Paulo Gonçalvès Richard G. Baraniuk* \\ Department of Electrical and Computer Engineering \\ Rice University, Houston, Texas, USA
}

\begin{abstract}
In this paper, we define a new set of tools for time-varying spectral analysis: the pseudo affine Wigner distributions. Based on the affine Wigner distributions of $J$. and $P$. Bertrand, these new time-frequency distributions support efficient online operation at the same computational cost as the continuous wavelet transform. Moreover, they take advantage of the proportional bandwidth smoothing inherent in the sliding structure of their implementation to suppress cumbersome interference components. To formalize their place within the echelon of the affine class of time-frequency distributions, we extend the definition of this class and introduce other natural generators.
\end{abstract}

\section{INTRODUCTION}

Time-frequency distributions (TFDs), which analyze signals in terms of joint time and frequency coordinates, have proven useful in a wide variety of fields. Most TFDs of current interest belong to either (or both of) Cohen's class [1] or the affine class $[2,3]$. While Cohen's class TFDs are covariant to time and frequency shifts of the signal, affine class TFDs feature an affine covariance to time and scale changes. This property makes affine distributions natural for a host of applications, including wideband radar and sonar, and selfsimilar signal analysis.

The simplest affine distribution is the continuous wavelet transform ${ }^{1}$

\footnotetext{
"E-mail addresses: gpaulo@rice.edu, richb@rice.edu. This work was supported by the National Science Foundation, grant no. MIP-9457438, the Office of Naval Research, grant no. N00014-95-1-0849, and the Texas Advanced Technology Program, grant no. TX-ATP 003604-002.

${ }^{1}$ Usually the wavelet transform is expressed as a function of a time variable $t$ and a scale variable $a$. Here we will use the reparametrization of scale as inverse frequency $a=f_{0} / f$ suggested in [3] and assume withour loss of generality that the center frequency $f_{0}$ of the wavelet $\psi$ equals $1 \mathrm{~Hz}$. Unless specified, bounds of integration nu from $-\infty$ to $+\infty$. We will also consider only analytic signals, for which $X(f) \equiv 0 \forall f<0$, with $X$ the Fourier transform of the time signal $x$.
}

$$
\begin{aligned}
D_{X}(t, f) & \equiv f^{-\frac{1}{2}} \int_{0}^{\infty} X(\nu) \Psi^{*}(\nu / f) e^{i 2 \pi \nu t} d \nu \\
& =f^{\frac{1}{2}} \int x(\tau) \psi^{*}(f(\tau-t)) d \tau
\end{aligned}
$$

It has the advantage of being a linear expansion of the signal onto a set of analyzing functions, yet its very linearity precludes desirable theoretical properties such as correct marginal distributions or perfect localization.

The quadratic affine Wigner distributions proposed in [2] are high resolution alternatives that have many desirable theoretical properties. Unfortunately, they also share two primary drawbacks. First, their nonlinearity results in copious interference terms in the time-frequency plane [4]. Second, due to their complicated formulation, efficient implementations suitable for long time series have not been developed for most of these distributions.

In this paper, we derive a set of (smoothed) pseudo affine Wigner distributions. Using the pseudo Wigner TFD as a guide, we introduce into the affine Wigner distributions a short-time window that not only controls the trade-off between localization and cross-component attenuation but also provides an efficient online computational algorithm.

The usual formulation of the affine class of TFDs, as the affine smoothing of the Wigner distribution with a kernel function [3], is not the most appropriate for the pseudo affine Wigner distributions. We will see that a more natural way of proceeding is to replace the Wigner distribution in this formulation with a set of canonical generating TFDs. We begin with a review of the affine Wigner distributions.

\section{AFFINE WIGNER DISTRIBUTIONS}

A TFD $P_{X}(t, f)$ of a signal $x$ is affine covariant if the distribution of the shifted and scaled signal $\frac{1}{\sqrt{\alpha}} x\left(\frac{t-t_{0}}{\alpha}\right)$ becomes $P_{X}\left(\frac{t-t_{0}}{\alpha}, \alpha f\right)$.

To overcome the limitations of the lincar wavelet transform, a broad class of bilinear distributions covariant to time and scale changes has been developed [2,3]. Among these distributions, an interesting sub-class is the class of affine 
Wigner distributions [2]

$$
\begin{gathered}
P_{X}^{(k)}(t, f) \equiv f \int \mu_{k}(u) X\left(\lambda_{k}(u) f\right) X^{*}\left(\lambda_{k}(-u) f\right) \\
\times e^{-i 2 \pi t f \xi_{k}(u)} d u
\end{gathered}
$$

with $k \in \mathbf{R}, \lambda_{k}(u)=\left(k \frac{e^{-z}-1}{e^{-k v-1}}\right)^{\frac{1}{k-1}}, \xi_{k}(u)=\lambda_{k}(u)-$ $\lambda_{k}(-u)$, and $\mu_{k}(u)$ a positive, continuous function. Proper choice of the function $\mu_{k}$ yields distributions that satisfy properties such as correct marginals, localization on powerlaw group delays, or unitarity. In addition to affine covariance, all affine Wigner distributions are covariant to a third unitary signal transformation [2].

For certain values of the index $k$, we obtain wellknown TFDs. The value $k=0$, corresponding to $\lambda_{0}(u)=\frac{-u}{e^{-u}-1}, \xi_{0}(u)=u$, and the choice $\mu_{0}(u)=$ $\left[\lambda_{0}(u) \lambda(-u)\right]^{\frac{1}{2}}\left(\frac{d \xi(u)}{d u}\right)^{\frac{1}{2}}$, yields the unitary Bertrand distribution

$$
\begin{aligned}
P_{X}^{(0)}(t, f) \equiv f \int & \mu_{0}(u) X\left(\lambda_{0}(u) f\right) X^{*}\left(\lambda_{0}(-u) f\right) \\
& \times e^{-i 2 \pi t f u} d u .
\end{aligned}
$$

Other well-known distributions in the affine Wigner class include the Wigner distribution $(k=2)$, the Flandrin $\mathrm{D}$ distribution $(k=1 / 2)$, the Unterberger distribution $(k=-1)$, and the Margenau Hill distribution (arithmetic mean of the limit cases as $k \rightarrow \pm \infty$ ).

\section{PSEUDO AFFINE WIGNER DISTRIBUTIONS}

\subsection{Definition}

Our derivation of the pseudo affine Wigner distributions relies on the strong analogy between Cohen's class and the affine class and is inspired by the pseudo Wigner distribution. The Wigner distribution

$$
W_{X}(t, f) \equiv \int x\left(t+\frac{\tau}{2}\right) x^{*}\left(t-\frac{\tau}{2}\right) e^{-i 2 \pi f \tau} d \tau
$$

has many attractive properties, but also drawbacks similar to the other affine Wigner distributions. The pseudo Wigner distribution was developed to overcome these difficulties. It is obtained from (4) by introducing a positive window function $h(\tau)$ both to allow efficient sliding computation and to smooth cross-components in the frequency direction. Convolution of the result with another window $g(t)$ smooths cross-components in the time direction. By defining a new window function $w(\tau)=\sqrt{h(2 \tau)}$, we can write the pseudo Wigner distribution as the convolution of the short-time Fourier transform $S_{X}(t, f) \equiv \int x(\tau) w^{*}(\tau-t) e^{-i 2 \pi f \tau} d \tau$ with itself [5]

$\widetilde{W}_{X}(t, f)=\int G(\nu) S_{X}(t, \nu) S_{X}(t, 2 f-\nu) e^{-i 4 \pi t(f-\nu)} d \nu$.
An analogous windowing procedure leads to the pseudo affine Wigner distributions. In contrast to the pseudo Wigner case, however, this windowing must be frequencydependent, to ensure that the resulting TFD remains affine covariant. As a result, the smoothing in frequency direction is proportional bandwidth, ${ }^{2}$ rather than constant-bandwidth as in the pseudo Wigner distribution.

By rewriting (2) in the time domain, windowing the signal using the short-time window $h$, and then posing $\psi(t)=$ $h(t) e^{i 2 \pi t}$, we can write the order $k$ pseudo affine Wigner distribution as

$$
\begin{array}{r}
\tilde{P}_{X}^{(k)}(t, f)=f \int \mu_{k}(u)\left[\int x(\tau) \psi^{*}\left[f \lambda_{k}(u)(\tau-t)\right] d \tau\right] \\
\times\left[\int x\left(\tau^{\prime}\right) \psi^{*}\left[f \lambda_{k}(-u)\left(\tau^{\prime}-t\right)\right] d \tau^{\prime}\right]^{*} d u \\
=\int \frac{\mu_{k}(u)}{\sqrt{\lambda_{k}(u) \lambda_{k}(-u)}} D_{X}\left[t, \lambda_{k}(u) f\right] D_{X}^{*}\left[t, \lambda_{k}(-u) f\right] d u,
\end{array}
$$

where $D_{X}(t, f)$ is the wavelet transform (1) based on the oscillating function $\psi$. Just as the pseudo Wigner distribution (5) is a convolution of the short-time Fourier transform of the signal with itself, the pseudo affine Wigner distribution is a generalized convolution of the wavelet transform with itself.

The pseudo affine Wigner distributions can be interpreted as sliding versions of the original affine Wigner distributions, and as a result, they are naturally suited for online operation with long signals. To construct a pseudo affine Wigner distribution, we simply compute the wavelet transform of the signal and then at each time perform the generalized frequency convolution defined by (7). The fast Mellin transform is a convenient tool for implementing this convolution efficiently $[6,7]$.

In the important special case of the unitary $P^{(0)}$ distribution, the special form for $\mu_{0}(u)$ cancels the $\left(\lambda_{k}(u) \lambda_{k}(-u)\right)^{-\frac{1}{2}}$ factor, leaving us with a much simpler expression for $\widetilde{P}^{(0)}[6]$. More generally, however, this approach allows us to approximate all affine Wigner distributions, even for unusual values of the index $k$ for which the algorithm proposed in [7] does not apply.

\subsection{Time-frequency smoothing interpretation}

The time windowing introduced in (6) acts as a proportional bandwidth frequency smoothing that suppresses interference components oscillating in the frequency direction. Compare, for example, the pseudo-Bertrand distribution $\widetilde{P}^{(0)}$ of Figure 1(b) with the unitary Bertrand distribution $P^{(0)}$ of Figure 1(a).

\footnotetext{
${ }^{2}$ Or constant- $Q$, with the $Q$ factor defined as analysis frequency over analysis bandwidth.
} 
To suppress interference terms oscillating in the time direction, we must smooth in that direction, or equivalently window the dual variable. The introduction of a low-pass function $G$ in (7) limits the integration with respect to $u$ (loosely speaking, the dual variable of the product $t f$ ), and thus performs proportional-bandwidth time smoothing of the TFD. We call the resulting distribution

$$
\begin{aligned}
\widetilde{P}_{X}^{(k)}(t, f) & =\int G(u) \frac{\mu_{k}(u)}{\sqrt{\lambda_{k}(u) \lambda_{k}(-u)}} \\
\times & D_{X}\left[t, \lambda_{k}(u) f\right] D_{X}^{*}\left[t, \lambda_{k}(-u) f\right] d u
\end{aligned}
$$

the smoothed pseudo affine Wigner distribution. (See Figure 1(c).) For $k=2$, this TFD coincides with the "affine smoothed Wigner distribution" of [3].

Even though the pseudo and smoothed pseudo affine Wigner distributions are smoothed versions of the affine Wigner distributions, they can still have resolution exceeding that of the scalogram, the squared magnitude of the wavelet transform. (See Figure 1(d).)

\section{KERNEL FORMULATION OF THE PSEUDO AFFINE DISTRIBUTIONS}

The pseudo affine Wigner distributions belong to the affine class, whose definition [3]

$$
P_{X}(t, f)=\iint_{0}^{\infty} W_{X}(\tau, \nu) \Pi\left(f(\tau-t), \frac{\nu}{f}\right) d \nu d \tau
$$

involves the Wigner distribution $W$ from (4). Unfortunately, this formalism turns awkward when one tries to derive an analytic form for the kernel $\Pi$ corresponding to a general pseudo affine Wigner distribution. In this section, we investigate a canonical formulation for the affine class of TFDs in which the kernels corresponding to the pseudo affine Wigner distributions have an easily identifiable, closed form.

For each $k \in \mathbf{R}$, we will introduce an alternative generator TFD $W^{(k)}$ that replaces $W$ in (9) and provides a natural framework for the pseudo affine Wigner distributions $\widetilde{P}^{(k)}$. With this new formalism, affine class TFDs can be written as

$$
P_{X}(t, f)=\iint_{0}^{\infty} W_{X}^{(k)}(\tau, \nu) \Pi^{(k)}\left(f(\tau-t), \frac{\nu}{f}\right) d \nu d \tau
$$

In Section 4.1, we find the appropriate generator TFD $W^{(k)}$ for each $k \in \mathbf{R}$. In section 4.2 , we identify the kernel corresponding to each smoothed pseudo affine Wigner distribution $\widetilde{P}^{(k)}$.

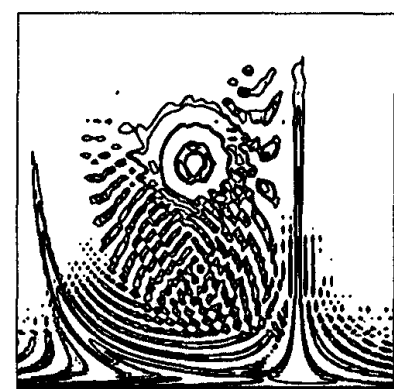

(a)

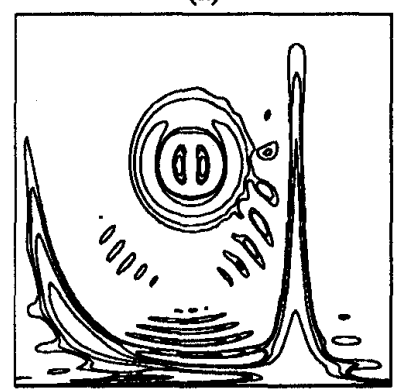

(c)

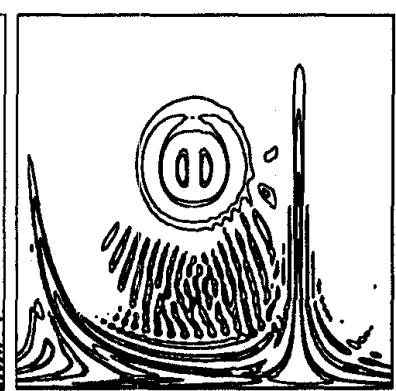

(b)

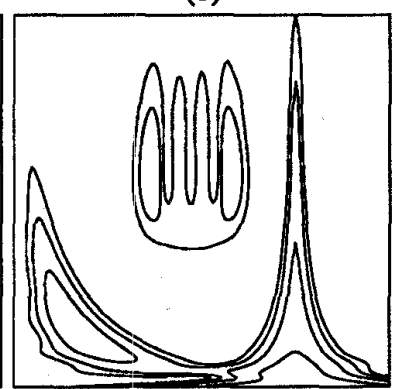

(d)
Figure 1. Time-scale distributions of a signal test composed of a hyperbolic chirp, a third-order Hermite function, and a Lipschitz singularity |t $\left.t_{0}\right|^{-0.1}$. Horizontal axis corresponds to time; vertical axis corresponds to frequency. (a) Unitary Bertrand distribution. (b) Pseudo Bertrand distribution computed with a Morlet wavelet $\psi$ of $Q=8$. (c) Smoothed pseudo Bertrand distribution computed with the same wavelet and a Gaussian window $G$ of $Q=1$. (d) Scalogram (squared magnitude of the wavelet transform) based on a Morlet wavelet of $Q=2$.

\subsection{Natural generators}

The most general form of an affine covariant TFD is given by $[2,3]$

$$
\begin{aligned}
P_{X}(t, f)=f & \int_{0}^{\infty} \int_{0}^{\infty} X(f \nu) X^{*}\left(f \nu^{\prime}\right) \\
& \times K\left(\nu, \nu^{\prime}\right) e^{i 2 \pi t f\left(\nu-\nu^{\prime}\right)} d \nu d \nu^{\prime}
\end{aligned}
$$

with $K(x, y)$ a two-dimensional distribution.

Fix $k \in \mathbf{R}$ and introduce into (11) the affine Wigner parametrization function $\lambda_{k}(u)$ by the change of variables $\nu=\theta \lambda_{k}(u), \nu^{\prime}=\theta \lambda_{k}(-u)$ with Jacobian $\partial\left(\nu, \nu^{\prime}\right) / \partial(\theta, u)=\theta \lambda_{k}(u) \lambda_{k}(-u)$

$$
\begin{aligned}
P_{X}(t, f) & =f \iint_{0}^{\infty} X\left(f \theta \lambda_{k}(u)\right) X^{*}\left(f \theta \lambda_{k}(-u)\right) \\
& \times K\left[\theta \lambda_{k}(u), \theta \lambda_{k}(-u)\right] e^{i 2 \pi t f \theta \xi_{k}(u)} \\
& \times \theta \lambda_{k}(u) \lambda_{k}(-u) d \theta d u .
\end{aligned}
$$

Since the function $\xi_{k}(u)$ defined in (2) is a one-to-one mapping from $\mathbf{R}$ to $\mathbf{R}$, the kernel $K$ can be reparametrized as

$$
K\left[\theta \lambda_{k}(u), \theta \lambda_{k}(-u)\right]=L\left[\theta, \theta \xi_{k}(u)\right]
$$


Taking into account the change of measure necessitated by this reparameterization, (12) can be rewritten as

$$
\begin{gathered}
P_{X}(t, f)=\iint_{0}^{\infty} f \theta \frac{d \xi_{k}(u)}{d u} X\left(f \theta \lambda_{k}(u)\right) X^{*}\left(f \theta \lambda_{k}(-u)\right) \\
\times\left[\frac{\lambda_{k}(u) \lambda_{k}(-u)}{\frac{d \xi_{k}(u)}{d u}} L\left[\theta, \theta \xi_{k}(u)\right] e^{i 2 \pi t f \theta \xi_{k}(u)}\right] d \theta d u .
\end{gathered}
$$

Finally, introducing the Fourier transform of $L$ with respect to $\theta \xi_{k}(u)$ and posing

$$
\begin{gathered}
\lambda_{k}(u) \lambda_{k}(-u)\left(\frac{d \xi_{k}(u)}{d u}\right)^{-1} L\left[\theta, \theta \xi_{k}(u)\right] e^{i 2 \pi t f \theta \xi_{k}(u)} \\
=\int \Pi(\theta, \gamma-t f) e^{-i 2 \pi \gamma \theta \xi_{k}(u)} d \gamma
\end{gathered}
$$

followed by the change of variables $f \theta=\nu, \gamma=f \tau$, leads us to the result (10) with the generator

$$
\begin{aligned}
W_{X}^{(k)}(\tau, \nu)=\nu \int & X\left(\nu \lambda_{k}(u)\right) X^{*}\left(\nu \lambda_{k}(-u)\right) \\
& \times e^{-i 2 \pi \nu+\xi_{k}(u)} \frac{d \xi_{k}(u)}{d u} d u
\end{aligned}
$$

Thus each generator is simply an affine Wigner distribution (2) with the special choice $\mu_{k}(u)=\frac{d \xi_{k}(u)}{d u}$.

Each different value of $k \in \mathbf{R}$ yields a different generator matched to the pseudo affine Wigner distributions $\widetilde{P}^{(k)}$. For $k=2$, we retrieve the Wigner distribution - the only unitary generator - and the usual affine class definition (9). For $k=-1$, we retrieve the active Unterberger distribution - the only generator that is time localized according to the definition of [2]. For $k=0$, we obtain a (nonunitary) Bertrand distribution with $\mu(u) \equiv 1$.

\subsection{Kernels for the pseudo affine Wigner distributions}

Using the results of the previous section, it is straightforward to derive the kernel $\Pi^{(k)}$ corresponding to each smoothed pseudo affine Wigner distribution $\widetilde{P}^{(k)}$. Again fixing $k \in \mathbf{R}$, we follow the four golden steps: (i) Use the Plancharel theorem to rewrite the right side of (6) in the frequency domain; (ii) Apply the same change of variables that took us from (11) to (12); (iii) Exploit the identity

$$
\begin{array}{r}
X\left(\nu \lambda_{k}\left[\xi_{k}^{-1}(-\theta / \nu)\right]\right) X^{*}\left(\nu \lambda_{k}\left[-\xi_{k}^{-1}(-\theta / \nu)\right]\right) \\
=\int W_{X}^{(k)}(\tau, \nu) e^{-i 2 \pi \tau \theta} d \tau ;
\end{array}
$$

(iv) Identify the result with the general expression (10). Doing this, we obtain the smoothed pseudo affine Wigner kernel

$$
\begin{aligned}
& \Pi^{(k)}(\tau, \nu)=\nu \iint G(u) \mu(u) \frac{\lambda_{k}(v) \lambda_{k}(-v)}{\lambda_{k}(u) \lambda_{k}(-u)} \\
& \quad \times \Psi\left(\nu \frac{\lambda_{k}(v)}{\lambda_{k}(u)}\right) \Psi^{*}\left(\nu \frac{\lambda_{k}(-v)}{\lambda_{k}(-u)}\right) e^{-i 2 x \tau \nu \xi_{k}(v)} d u d v .
\end{aligned}
$$

It is interesting to note that setting $G(u)=\delta(u)$ reduces (8) to the scalogram. In this case, (10) simplifies to

$$
\left|D_{X}(t, f)\right|^{2}=\iint_{0}^{\infty} W_{X}^{(k)}(\tau, \nu) \widehat{W}_{\Psi}^{(k)}\left(f(\tau-t), \frac{\nu}{f}\right) d \nu d \tau .
$$

Here, $\widehat{W}^{(k)} \equiv \Pi^{(k)}$ is the passive form of the generator $W^{(k)}$. While the active form $W^{(k)}$ is nonunitary in general, it cooperates with its passive form to produce an isometrylike relation [2]

$$
\iint_{0}^{\infty} W_{X}^{(k)}(t, f) \widehat{W}_{Y}^{(k)}(t, f) d f d t=|\langle X, Y\rangle|^{2} .
$$

Of all the generators, only for $k=2$ (the Wigner distribution) do we have $\widehat{W}^{(k)}=W^{(k)}$. In this case, (14) coincides with the Wigner-based scalogram formulation of [3].

\section{CONCLUSIONS}

Although the affine Wigner distributions have many attractive properties, interference terms and lack of efficient implementations have limited their impact on time-varying signal analysis. By overcoming some of their limitations, the pseudo and smoothed pseudo affine Wigner distributions should open up new application areas to these powerful tools. In particular, the flexible windowing scheme underlying these new TFDs allows a continuous transition in smoothing between affine Wigner distributions and scalograms.

The introduction of alternative generators for the affine class of TFDs simplifies the kernel formulation of the pseudo affine Wigner distributions. In addition, the concept of alternative generators should aid in the analysis and design of new affine distributions. ${ }^{3}$

\section{REFERENCES}

[1] L. Cohen, Time-Frequency Analysis. Englewood Cliffs, NJ; PrenticeHall, 1995.

[2] J. Bertrand and P. Bertrand, "A class of affine Wigner functions with extended covariance properties," J. Math. Phys., vol. 33, pp. 25152527, July 1992.

[3] O. Rioul and P. Flandrin, "Time-scale energy distributions: A general class extending wavelet transforms," IEEE Trans. Signal Processing, vol. SP-40, pp. 1746-1757, July 1992.

[4] P. Flandrin and P. Gonçalvès, "Geometry of affine time-frequency distributions," Appl. Comp. Harmonic Anal., Ian. 1996. To appear.

[5] L. Stanković, "A method for time-frequency analysis," IEEE Trans. Signal Processing, vol. SP-42, pp. 225-229, Jan. 1994.

[6] P. Gonçalvès and R. G. Baraniuk, "A pseudo-Bertrand distribution for time-scale analysis," IEEE Signal Processing Letters, 1996. To appear.

[7] J. P. Ovarlez, J. Bertrand, and P. Bertrand, "Computation of affine time-frequency distributions using the fast Mellin transform," in Proc. IEEE Int. Conf. Acoust., Speech, Signal Processing - ICASSP '92, (San Francisco, CA, USA), pp. V117-V120, 1992.

\footnotetext{
${ }^{3}$ The authors wish to thank J. Bertrand, H. Feichtinger, and A. Sayeed for their insightful comments.
} 